\title{
Gender differences in the rate of motor activity of senior (older) preschool children
}

\author{
Lyudmila Voloshina, Victor Kondakov, Evgeniya Kopeikina*, Olga Galimskaia, Elena Arseenko
}

Belgorod State National Research University, 308015, Belgorod, Russia

\begin{abstract}
The relevance of the research is determined by the search for ways to improve the content of physical education of preschool children and increase its effectiveness in gender-based motor activity. Goal: to identify gender differences in older preschoolers' motor activity. Research materials: the study involved preschool children aged 5-7 years who attend pre-school in Belgorod. Research methods: study, analysis and comparison of scientific publications of domestic and foreign scientists on the problem of research, pedometer, heart rate monitoring, timing, statistical processing of the results. Results: the features of motor activity of boys and girls aged 5-7 years were studied in such indicators as daily and weekly volume in steps, intensity in organized forms and independent activity. Conclusions: gender differences in the size, structure, and content of motor activity are most noticeable at the age of five. There are no gender differences in motor activity of preschool children aged 6-7 years. Significant gender differences in children aged 5-7 years are only in indicators of motor activity recorded on weekends, outside of classes. The value of motor activity of children aged 5-7 years corresponds to the age norm of average daily motor activity.
\end{abstract}

\section{Introduction}

Optimal motor activity is a powerful tool for improving health, increasing the physical condition of the child, and the well-being of his / her psychoemotional state. There is an urgent need for pedagogical support of the optimal motor regime of a growing person. With due regard for individual age and gender characteristics it will help to solve this important pedagogical task. This makes it necessary to study in-depth both individual and gender characteristics of children's motor activity. Age and individual characteristics of preschool children motor activity are determined by the conditions of their organization. By the older preschool age, children who have a high need for physical activity cannot always implement it at the proper level. At the same time, independent motor activity of children at this age is limited by the created conditions in kindergarten and family. An increase of educational classes with a predominance of static poses, as well as increasing cognitive interests of children (computer games, designing, watching TV shows, etc.) lead to a decrease in motor activity. This is noted in the works of Komkov A. G, Antipov E. V. (2003), Lubysheva L. I. (1997) and foreign authors Laukkanen A, et al. (2018), Ward S, et al. (2018), Currie C et al., (2012) [1-5]. Detailed analysis of scientific publications of foreign scientists Hacke C, et al. (2019), Niemisto D, et al. (2019), Zeng N, et al. (2019), Maatta S, et al. (2019) indicates a high interest in optimizing motor activity and making programs, taking into account social factors that characterize the educational process in various countries of the world [6-16].

The aim of the study was to identify gender differences in the indicators of senior preschool children motor activity.

\section{Materials and methods}

The study involved 91 boys and 77 girls aged 5-7 years from kindergartens No. 57, 64, 69 Belgorod, Russia. Research methods: study of scientific publications of domestic and foreign scientists on the problem of research, pedometer, heart rate monitoring, timing, statistical processing of the results.

\section{Results and discussion}

The most noticeable differences between boys and girls at the age of five are in the motor activity indicators during independent activity. It is due to the different content of their play activities. Boys are dominated by mobile games (football, "Tag", etc.). At the same time, girls ' play activities are characterized by more calm, story-role-playing games ("Daughters-mothers", "shop", "School", etc.). The greatest value of motor activity of preschool children aged 5 years is during walks in preschool. It reaches $1185.29 \pm 133.47$ steps per hour in boys, and $1098.86 \pm 143.91$ steps per hour in girls. (Fig. 1.)

The average volume of motor activity in 5-year-old boys during wakefulness is $762.77 \pm 67.70$ steps per hour, and in

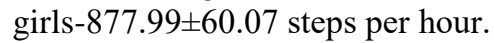

\footnotetext{
* Corresponding author: evgeniyakopeikina@yandex.ru
} 


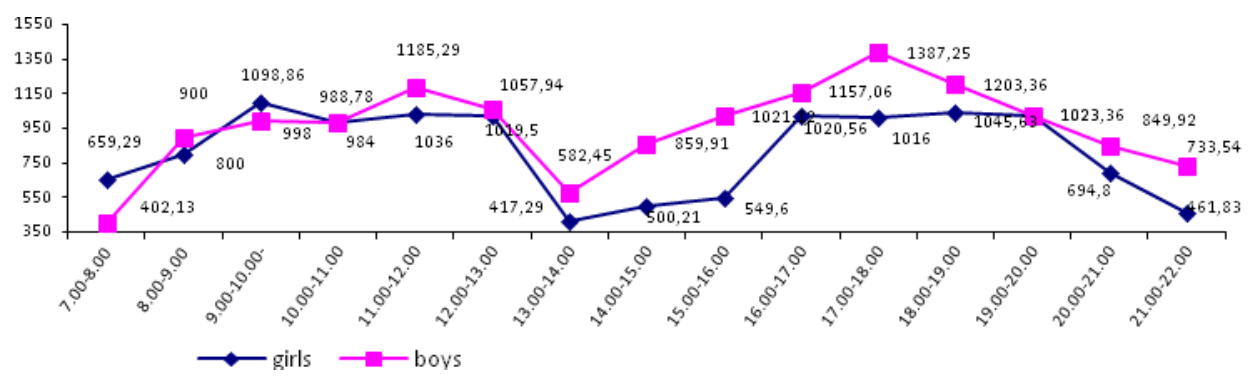

Fig. 1. Motor activity dynamics of 5 years old children (waking time).

Motor activity of preschool children aged 6 years is more meaningful and diverse than the activity of 5 years old children. The mastering new motor skills encourages a high interest in outdoor games and relay races in 6 years old children, both boys and girls. Children of this age enjoy using whole arsenal of motor actions.

There are no sharp differences in the content of motor activity of boys and girls, which is typical for 5-year-old preschoolers. The characteristic feature of the 6 years old preschoolers game activity is the association in small groups to perform various physical exercises and outdoor games.

The greatest motor activity in boys aged 6 years is during walks in kindergarten. It reaches $1213.95 \pm 217.74$ steps per hour. Girls do $1306.40 \pm 214.78$ steps per hour (Fig. 2.). A comparative analysis showed that activity did not increase significantly in boys, while in girls there was a significant increase, due to changes in the structure and content of independent motor activity.

The average volume of motor activity in boys aged 6 years during wakefulness is $805.47 \pm 52.94$ steps per hour, and in girls-868.69 \pm 68.95 steps per hour.

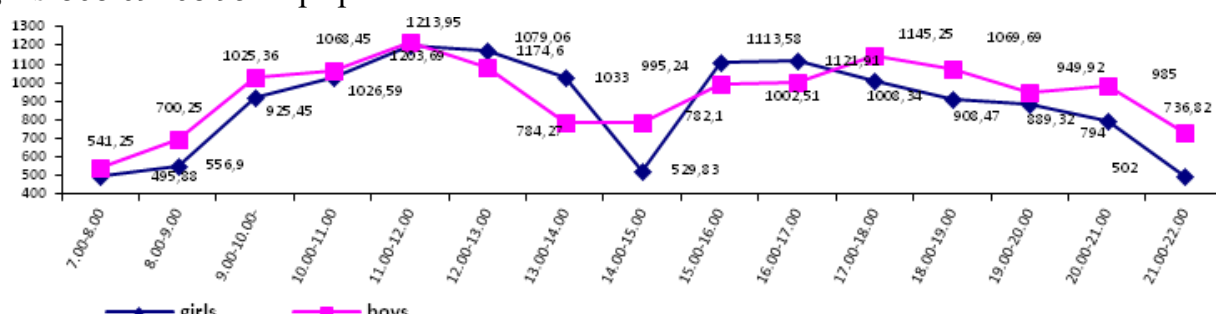

Fig. 2. Motor activity dynamics of 6 years old children (waking time).

There is a significant increase in the arsenal of motor skills and abilities of preschool children aged 7 years compared to the previous age groups. Indicators of motor activity increase due to the introduction of children to organized classes in sports sections (12\% of the surveyed) and independent motor activity. There are no gender differences in the preference for certain types of motor activity in this age period. Both boys and girls show a fairly stable interest in the same outdoor games. At this age, children prefer outdoor games. There is no noticeable increase in motor activity in both boys and girls. The greatest motor activity in boys aged 7 years is during walks in kindergarten. It reaches $1288.24 \pm 259.14$ steps per hour, and in girls the same indicator is $1338.80 \pm 266.05$ steps per hour (Fig. 3.).

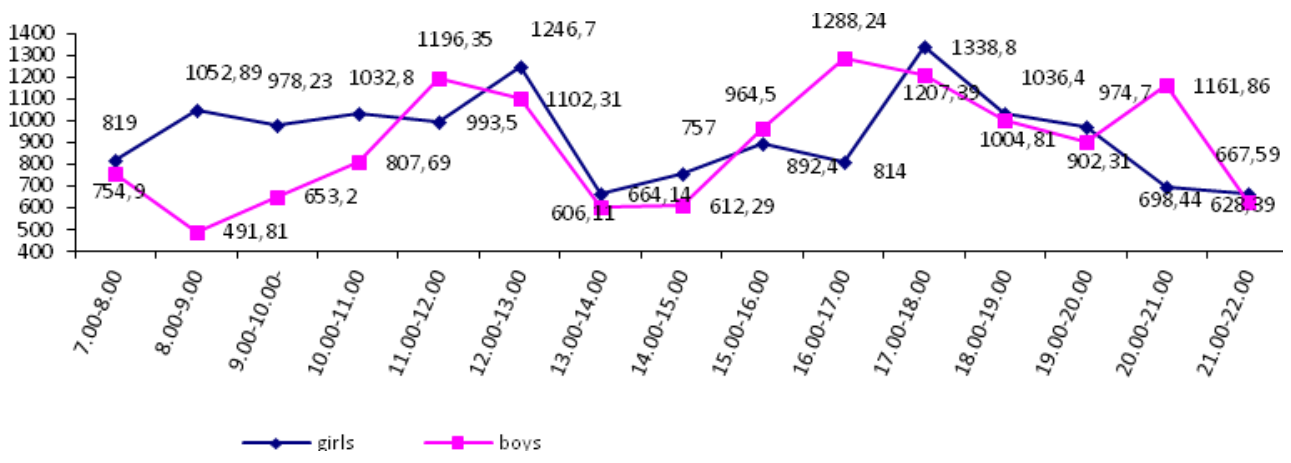

Fig. 3. Motor activity dynamics of 7 years old children (waking time).

The average volume of motor activity of 7-year-old boys during wakefulness is $896.07 \pm 70.34$ steps per hour, and in girls-937.47 \pm 53.49 steps per hour.

The motor activity of preschool children aged 5-7 years is distributed unevenly throughout the day (Fig. 1-3). Preschool children aged 5-7 years have the greatest motor activity during walks, organized physical education classes in preschool sports sections. They have low motor activity during the staying in the room. During the day, 2 peaks of 
motor activity are registered: from 10.00 to 12.00 and from 16.00 to 19.00 (Fig. 4). This dynamics of motor activity is due to the kindergarten's day order, which is based on the physiological characteristics of the child's body.

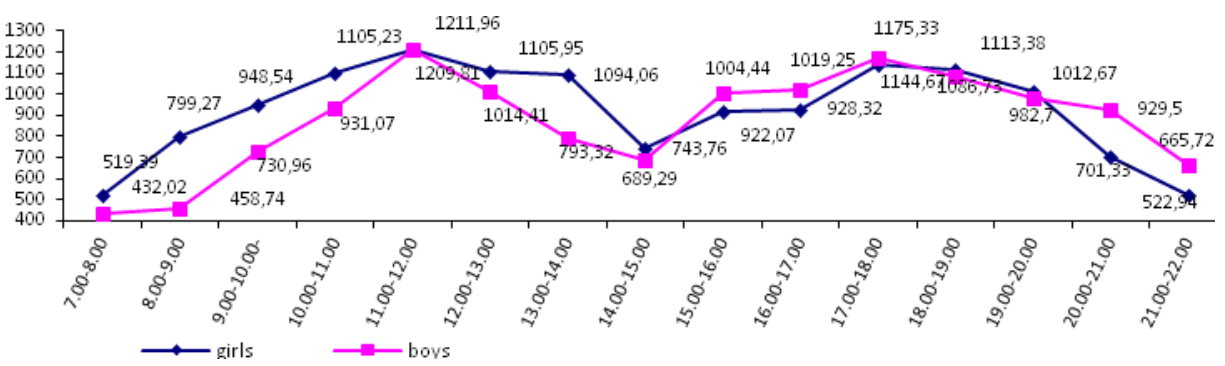

Fig. 4. Average daily dynamics of motor activity intensity in children aged 5-7 years (waking time).

Statistical analysis of weekly pedometry of preschool children aged 5-7 years (waking time) allowed us to estimate the average indicators of motor activity intensity, which in boys is $631.74 \pm 50.37$ steps per hour, and in girls $925.82 \pm 21.41$ steps per hour. The dynamics of the average weekly pedometry of preschool children aged 5-7 years shows that on working days the motor activity intensity of boys and girls is equal. On weekends the intensity of boys is significantly lower than that of girls. The highest intensity of motor activity in boys is observed on Friday, and the lowest on Sunday. At the same time, girls have the highest intensity of motor activity on Saturday, and the lowest-on Monday.

The weekly volume of motor activity of preschool children aged 5-7 years is $13123.29 \pm 590.34$ steps per day for boys, and $13873.53 \pm 446.44$ steps per day for girls. (Fig. 6). Analysis of the data obtained and comparison with the age norm This indicates that both boys and girls meet the age norm of average daily motor activity (11-15 thousand steps per day (according to A. G. Sukharev)). Significant gender differences in children aged 5-7 years are in indicators of motor activity on weekends, outside of organized classes in preschool.

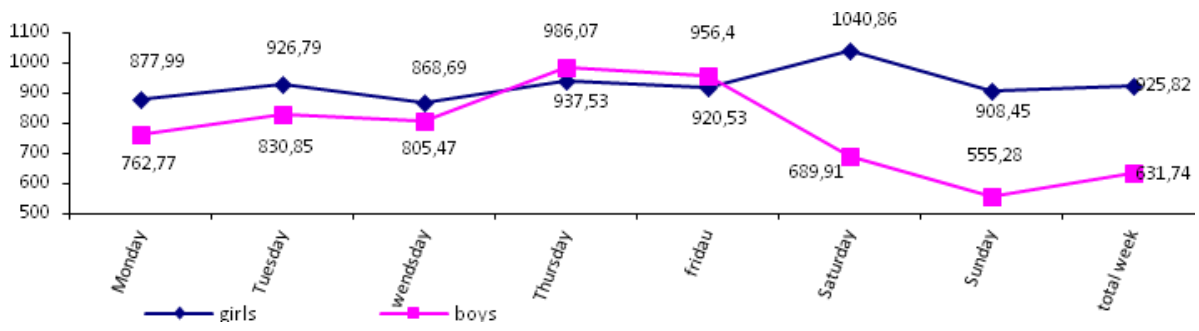

Fig. 5. Weekly dynamics of motor activity intensity in children 5-7 years old (waking time).

The highest motor activity in boys is on Friday, and the lowest on Tuesday. At the same time, girls have the highest motor activity on Saturday, and the lowest-on Sunday. This dynamic is explained by the fact that most of the children have trainings in sports sections on these days (for boys - Friday, and for girls - Saturday).

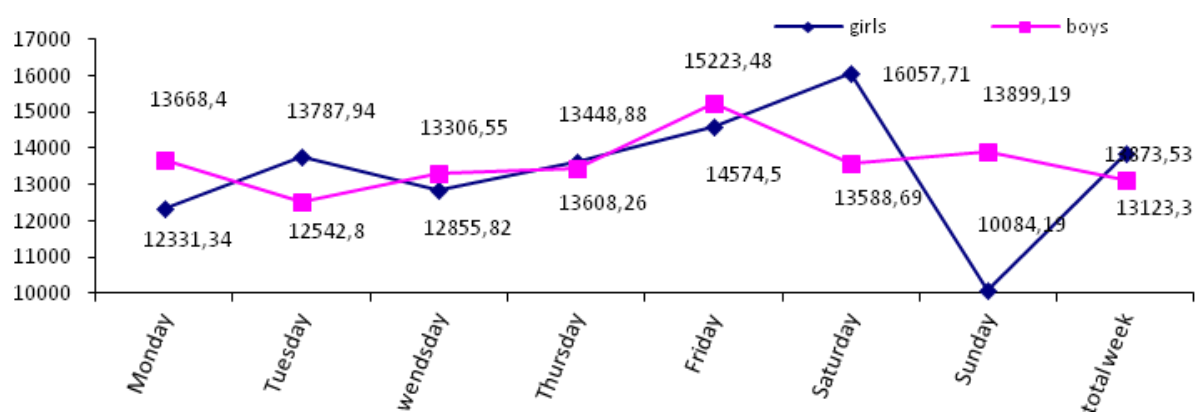

Fig. 6. Weekly dynamics of daily motor activity in children aged 5-7 years.

According to Russian and foreign scientists, the main reasons for reducing motor activity in preschool age are sociopedagogical determinants. [10,12,13,17].

Grebneva V. V. and Sadovsky M. V. (2020) in their work point to the need to take into account not only gender differences on cultural grounds, but also gender differences by biological type (masculine (male), feminine (female) and androgynous (mixed)) [18].

Scientific research by V. V. Karikh and O. U. Zaitseva proved the need to take into account gender differences in older preschool children in physical education classes. They also draw attention to the fact that the educational environment should be aimed at overcoming gender stereotypes in the joint educational activities of a teacher with 
preschool children and managing the formation of gender identity in physical education classes in preschool education organizations [19].

Pellegrini, Anthony D.; Long, Jeffrey D.; Roseth, Cary J.; with co-authors (2007) studied the gender features of children's motor activity in terms of the interactive role of biology and socialization. They concluded that children aged 5-7 years are characterized by gender differences in the choice of motor activity types. Such differences were noted in 5-year-old children [20].

In a number of studies, the problem of reducing motor activity and muscle effort is justified by social factors $[3,4,6,7,8,9]$. This is most often associated with the realities of modern civilization: physical inactivity, changing interests, new forms of leisure (computer games, watching TV, etc.) [1,2,5].

There are gender differences in the size and structure of motor activity in older preschoolers. Gender differences are most clearly manifested in preschool children aged 5 years, and in 6-7 years there is a tendency to gradually smooth out the boundaries [21].

\section{Conclusions}

There is a close relationship between quantitative indicators of motor activity of children aged 5-7 years with the nature and content of their activities during their stay in preschool.

1. Gender differences in the size, structure and content of motor activity are most noticeable at the age of five.

2. Gender differences, both in the weekly daily volume of motor activity, and in the preference for certain types of motor activity in preschool children 6-7 years old are not observed.

3. Significant gender differences in children aged 5-7 years are observed only in indicators of motor activity recorded on weekends, outside of organized classes in preschool.

4. The value of motor activity of children aged 5-7 years corresponds to the age norm of average daily motor activity.

Our research is not an exhaustive solution to this problem. Additional studies with a large sample size and duration are required.

\section{Acknowledgment}

The article was supported by the grant of the Russian State Scientific Fund No. 19-013-00173 “A comprehensive study of the motor activity of a growing person under a variable system of physical education".

\section{References}

1. A.G. Komkov, E.V. Antipova, Theory and practice of physical culture 3, 5-8 (2003)

2. L.I. Lubysheva, Theory and practice of physical culture 6, 10-15 (1997)

3. A. Laukkanen, D. Niemisto, T. Finni, M. Cantell, E. Korhonen, A. Saakslahti, Scandinavian Journal of Medicine \& Science in Sports 28(12), 2691-701 (2018)

4. S. Ward, A.F. Chow, M.L. Humbert, M. Belanger, N. Muhajarine, H. Vatanparast, et al. Evaluation and Program Planning 68, 90-8 (2018)

5. C. Currie et al., eds. Copenhagen, WHO Regional Office for Europe 6 (2012)

6. C. Hacke, S. Ketelhut, U. Wendt, G. Muller, C. Schlesner, K. Ketelhut, Scandinavian Journal of Medicine \& Science in Sports 29(5), 742-52 (2019)

7. D. Niemisto, L.M. Barnett, M. Cantell, T. Finni, E. Korhonen, A. Saakslahti, Scandinavian Journal of Medicine \& Science in Sports 29(5),753-65 (2019)

8. N. Zeng, S.L. Johnson, R.E. Boles, L.L. Bellows, Journal of Sport and Health Science 8(2), 122-9 (2019)

9. S. Maatta, J. Gubbels, C. Ray, L. Koivusilta, M. Nislin, N. Sajaniemi, et al, Early Childhood Research Quarterly 47, 39-48 (2019)

10. D. Aivazidis, F. Venetsanou, N. Aggeloussis, V. Gourgoulis, A. Kambas, Journal of Physical Activity \& Health 16(3), 184-90 (2019)

11. M. Duncan, A. Cunningham, E. Eyre, European Physical Education Review 25(1), 221-35 (2019)

12. A.C. Engel, C.R. Broderick, N. van Doorn, L.L. Hardy, B.J. Parmenter, Sports Medicine 48(8), 1845-57 (2018)

13. A. Johnstone, A.R. Hughes, A. Martin, J.J. Reilly, Bmc Public Health 18, (2018)

14. M.H. Quan, H.B. Zhang, J.Y. Zhang, T. Zhou, J.M. Zhang, G.G. Zhao, et al, Journal of Clinical Medicine 7(5), (2018)

15. Y. Fu, R.D. Burns, N. Constantino, P. Zhang, Games for Health Journal 7(5), 335-40 (2018)

16. J. Jascenoka, F. Walter, F. Petermann, F. Korsch, S. Fiedler, M. Daseking, Kindheit Und Entwicklung 27(3), 142-52 (2018)

17. L.N. Voloshina, V.L. Kondakov, A.A. Tretyakov, E.N. Kopeikina, M. Cretu, V. Potop, Pedagogics, psychology, medicalbiological problems of physical training and sports 22(2), 114-119 (2018) DOI:10.15561/18189172.2018.0208

18. V. V. Grebneva, M. V. Sadovsky, Modern science-intensive technologies 1, 63-67; (2020)

19. A.S. Kuznetsov, Z.M. Kuznetsova, Russian Journal of Physical Education and Sport, 14(4), 5-7 (2019). DOI: 10.14526/20704798-2019-14-4-5-7 
20. A.D. Pellegrini, J.D. Long, C.J. Roseth, C.M. Bohn, \& M. Van Ryzin, Journal of Comparative Psychology 121(3), 282-289 (2007) DOI:10.1037/0735-7036.121.3.282

21. L.N. Voloshina, V.L. Kondakov, E.N. Kopeikina, \& O.G. Galimskaia, Humanities \& Social Sciences Reviews 7(6), 221-226 (2019) DOI:10.18510/hssr.2019.7641. 